\title{
Statistical method for the evaluation of railway systems modifications
}

\author{
M. Chandesris \\ SNCF Direction de la Recherche et de la Technologie, \\ Unité de Recherche "Génie Décisionnel Appliqué”, Paris, France
}

\begin{abstract}
Railway production plans daily face random events. Therefore, before setting up modifications for the railway system (changing the network, new signalling system, new timetable, etc.), its robustness to stochastic events must be estimated.

Tests cannot actually be carried out, which is why the SNCF uses a traffic simulator developed by its research department. Up to now, the timetable robustness has been estimated by considering a few small incidents and their simulated consequences on train delays.

The new approach we are working on consists of taking into account the stochastic dimension in the evaluation of robustness. The methodology is divided into three parts:

- Identifying randomness to be taken into account when evaluating robustness

- Studying the robustness of the railway system:

o determine experimental design

- carry out simulations

- aggegrate results

- Evaluating the lack of precision in the results
\end{abstract}

The aim of this project is to develop the required statistical methods and to design a decision tool following the three steps above. It will help the infrastructure managers to evaluate, in a statistical manner, the robustness of various railway system configurations.

\section{Timetable robustness and traffic simulator}

Railway production plans daily face random events. Therefore, before setting up modifications for the railway system (changing the network, new signalisation 
system, new timetable, etc.), its robustness to stochastic events must be estimated.

This paper is divided into three parts:

- Introduction to timetable robustness and the use of traffic simulator

- Proposition for methodology to evaluate robustness of timetable

- Examples of comparing different alternatives for a railway system thanks to the tool.

This first section introduces timetable robustness, simulation model and stochastic dimension of the problem.

\subsection{Timetable robustness}

Railway production daily faces random events that perturb traffic quality. When evaluating new production plans, these random events have to be included in the analysis of quality.

Such an approach allows introducing more elements when evaluating different modifications for the railway system (increase in traffic density, modification of signalling systems, etc.). Costs, theoretical capacity indicators may be available a priori, directly computable by looking at proposals. But indicators of service quality such as regularity involve randomness phenomena, which is more difficult to take into account in computations.

Timetable robustness is the capacity of a timetable to resist to little perturbations. (We concentrate on little perturbation, considering situations without active regulation.)

\subsection{Simulation model}

Actual tests cannot be carried out due to several reasons: security, costs, time, etc. Furthermore, the railway system is too complex to be solved by analytical solution. Simulation techniques provide a solution to these problems. The SNCF research department has developed a numerical simulator of the railway system [3]. The simulation model is briefly described.

The railway system such as it is described in SISYFE (SImulateur de SYstème FErroviaire), is comprised of two main subsystems that interact:

- the rolling stock with its driver and the fixed installations of the rail network;

- $\quad$ the human action of men outside this system (signalmen or traffic managers) classified as its environment.

The simulation allows, by iterative calculations, to simultaneously reproduce the changing state of signals and the evolution of the vehicles. The railway system is modelled by a discrete events system. At every moment, the state of the system is determined by:

- former states;

- $\quad$ actions of the environment;

It is a quasi-deterministic system because relation of transition checks consistency conditions. Propagation of state changes generates conditions that will characterise the following states. 
Such a tool allows studies of the timetable with several hundred of trains during hours.

The choice of a simulation tool is not restrictive; other simulation tools could be used instead of SISYFE. A lot of simulation tools have been recently developed by of for railway companies. But, nowadays, the key question is no longer "how to build a traffic simulator and how to choose a simulation model?" but "which scenarios have to be simulated into the simulator?".

\subsection{Stochastic dimension}

The natural randomness of the production process has to be taken into account. The Monte Carlo methods [4] provide approximate solutions to such problems by performing statistical sampling experiments on a computer.

The idea is to sample a perturbed timetable in an efficient way, then put them into the simulation tool, and then analyse the way perturbations are absorbed and finally aggregate results to make statistical conclusions.

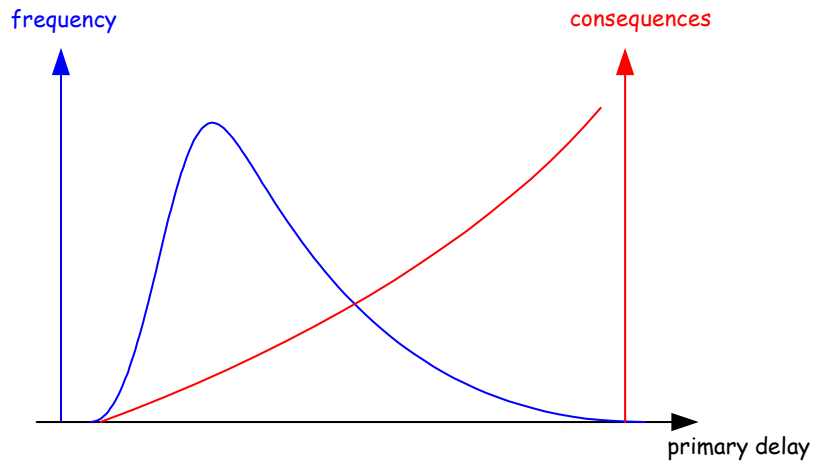

Figure 1: Duration of primary delay occurrence frequency and operational consequences.

\section{Evaluation of timetable robustness - methodology}

This second section describes how to:

- identify randomness;

- study robustness by simulation;

- evaluate a lack of precision in the results.

\subsection{Identify randomness}

The first task is to identify random events that would be taken into account during the analysis. Two points of view should be considered:

- incidents that occur during actual exploitation of the system are listed in databases from the feedback. Those databases allow the classification of incidents by type and to study their occurrence frequency; 
- incidents that do not cause any perturbation to the system are not to be included in the analysis, in order to optimise the use of simulation. A first set of simulated incidents makes it possible to identify characteristics of the incidents that cause operational consequences.

By studying these two aspects of random events that occur during railway production, we can -in an optimal way-determine which incidents should be taken into account during evaluation of robustness.

\subsection{Study robustness of the railway system}

\subsubsection{Determine experimental design}

Simulations have to be carefully planned, in order to best use the simulation time. The theory of experimental design [1] contains very useful techniques. The full design is divided into two parts:

1. the main design produces a vary hypothesis for the railway system (configuration parameters). At this step, we must set what we want to test: new timetables with increasing traffic density, new signalling system, new infrastructures, etc.

2. the second design varies the characteristics of incidents. Several characteristics of incident may be taking into account:

o type of the incident;

- delayed departure;

- $\quad$ speed limitation for a train or a portion;

- unexpected stop;

- emergency stop;

○ primary delay;

- place where the incident occurs;

- profile of the line where the incidents occurs;

- speed of the train when the incident occurs;

- space margin of the train when the incident occurs;

o rolling stock of the first train affected;

The building of the incidents design is helped by the former step "identify randomness".

Each configuration will be tested on the same types of incidents (but not necessarily the same incidents). When joining the experimental designs for configuration and for incidents, a cross-over design is obtained:

Establishing the experimental design is a key step: at this moment we optimise the compromise between:

- number of simulations;

- representative of incidents;

- $\quad$ accuracy of the results. 


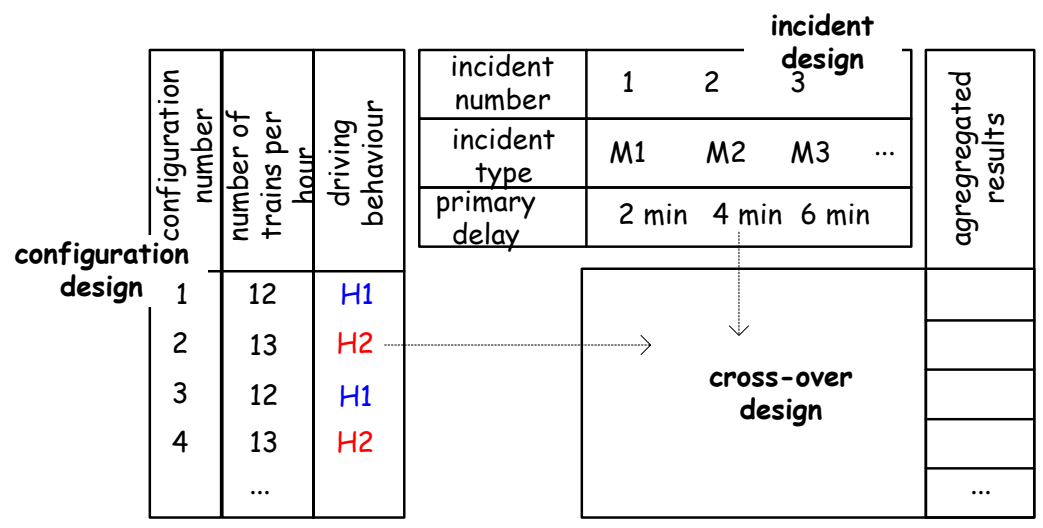

Figure 2: $\quad$ Example of experimental design.

\subsubsection{Carry out simulations}

SISYFE is based on a very detailed model of the railway system: numerous different configuration hypothesis and types of incidents may be tested. It provides hypothesis trees in order to automatically launch numerous sets of simulations with various hypotheses. We have been working on parallel autorepartition in order to save time when simulating hundred even thousand simulations.

For each pair (configuration, incident) simulated, SISYFE provides the transport plan "as if" it occurred in such conditions. The life for each train is available: where it is along its path and at what time. Secondary delays are deduced by comparison with theoretical schedule. A lot of numerical and graphics indicators are proposed to analyse this result.

\subsubsection{Aggregate results and analysis}

The next sep is to aggregate results. Two ideas should be kept in mind while the simulation results are aggregated:

- all incidents have to be aggregated by configuration in order to compare configurations between each other.

- $\quad$ incidents must be weighted (by frequency) in order to obtain actual quality indicator values;

To test significance of parameter variations such as traffic density, statistical techniques are used. The main one is variance analysis; the main concepts are explained below.

2.2.3.1 Variance analysis Take the example of the comparison of two configurations for the system. The first configuration is S0, generally the actual railway system configuration. By simulating incidents with this configuration, the distribution of the quality indicator studied is obtained. We write it SO(I) $->\mathrm{R}$ $+\varepsilon$. With $90 \%$ chance, the quality indicator is between $\mathrm{R}-\varepsilon$ and $\mathrm{R}+\varepsilon$.

When testing an improvement of the actual configuration, we test $\mathrm{S} 1=\mathrm{S} 0+\Delta \mathrm{S}$. The new quality indicator is $\mathrm{R} 1$. $[\mathrm{S} 0+\Delta \mathrm{S}](\mathrm{I})->\mathrm{R} 1+\varepsilon=\mathrm{R}+\Delta \mathrm{R}+\varepsilon$. 


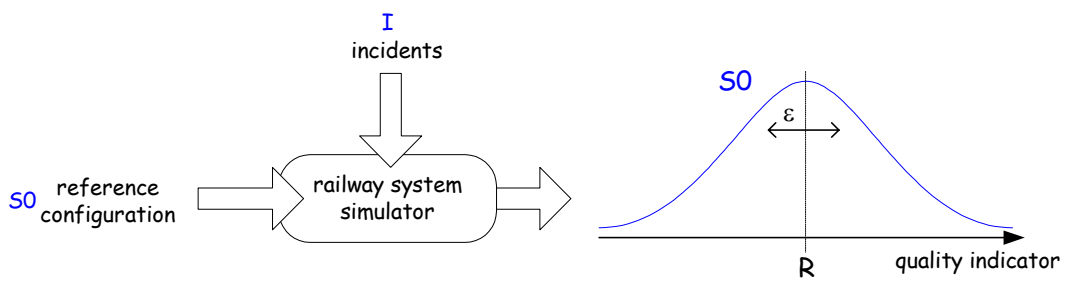

Figure 3: Distribution of the quality indicator obtained by simulations.

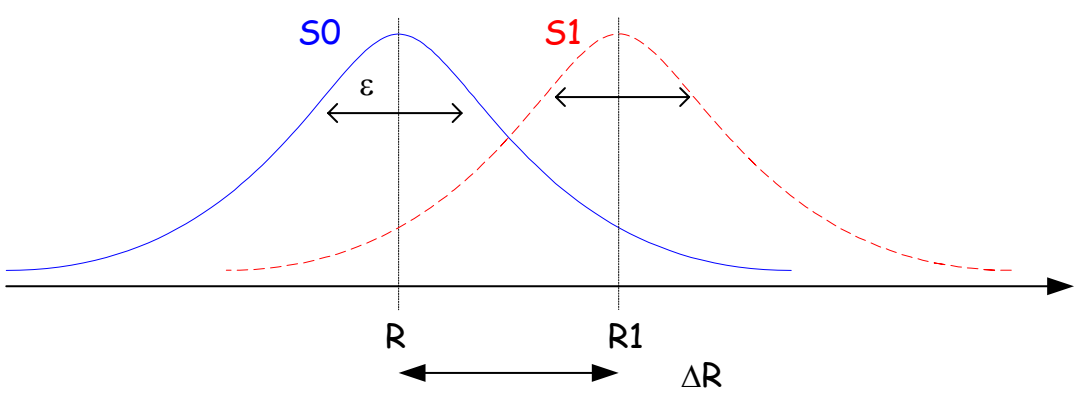

Figure 4: Comparison between two distributions of quality indicator.

The objective is to identify if the modification of the railway system is significant, whether is it worth implementing the modification? The two situations are judged by comparing the variability term and the quality improvement $\Delta \mathrm{R}$. The modification is significant if $\Delta \mathrm{R}>\varepsilon$.

\subsection{Evaluating lack of precision in the results}

Another crucial point is the estimation of the error of the present method. When announcing a rate of regularity under hypothesis of configuration, a confidence interval is added. It is more pragmatic and more interesting to provide piece of information of type: the rate of regularity is between $\mathrm{R} 1$ and $\mathrm{R} 2$ than to provide only R.

This is illustrated by the following example: we wish to increase the number of trains per hour. But, if the number of trains per hour is increased, delays are spread more easily because trains are closer together. So we want to test if this new delay can be compensated for by changing the trains are driven. Two hypotheses, $\mathrm{H} 1$ and $\mathrm{H} 2$, have to be tested.

At first glance at Figure 5, if the actual situation is 12 trains per hour with $\mathrm{H} 2$ (red in dot line), the number of trains per hour can be increased without losing regularity if the driving behaviour changes to $\mathrm{H} 1$ (blue in plain line). 

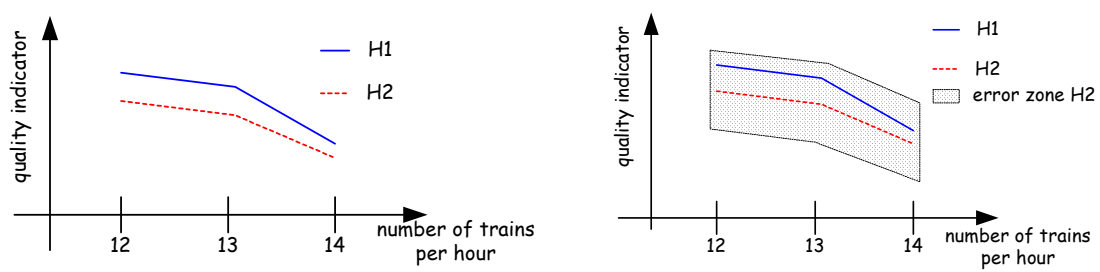

Figure 5: Interest of providing confidence interval.

But, considering the confidence interval of the evaluation process, this special case cannot be concluded, because the error made on the evaluation is greater than the difference between the two hypotheses.

If different hypotheses have to be compared in order to take investment decision, the gap between indicators of each hypothesis have to be bigger than the precision of the process. Otherwise, we cannot be sure of results of the decision.

The lack of precision in the process of measurement of robustness comes from three main sources:

1. in the feedback databases, the estimation of occurrence frequency of incident types may be biased;

2. a simulation model is a simplification of the actual world;

3. only a finite number of incidents are simulated.

Finally, the lack of precision may be evaluated by:

- standard parametric techniques such as confidence interval if normality assumptions are fulfilled;

- $\quad$ non parametric techniques, bootstrap may be used for example [2].

We have been working on both methods to obtain the smallest error term.

\section{Compare different alternatives for a railway system}

Such a methodology provides a decision indicator for service quality. In this section, we expose some examples of improvements of railway system that could be evaluated trough this procedure. Then the tool that has been developed is briefly presented.

\subsection{Examples of studies}

\subsubsection{Adding infrastructure}

Such tool may be used to evaluate the contribution of a new infrastructure. For example, the regularity with and without a third track could be compared. Then the quality contribution of this new track could be compared to the economic costs. 


\subsubsection{Increasing traffic density}

A first study on the high-speed line between Paris and Lyon has been done, taking into account about 5000 simulations. The influence of two parameters have been tested:

- $\quad$ the number of trains per hour : $12,13,14$ or 15 trains/hour;

- $\quad$ two driving behaviour parameters: A1, A2 and L1, L2.

\subsubsection{Modifying signalling and / or routing systems}

Another study involves an interconnection project in Paris and suburbs. Hundreds of trains of different speed are involved. We want to evaluate the contribution of the ETCS 2 signalling system with manual or automatic driving. Different systems of regulation have to be tested too.

\subsection{A statistical tool to help decision makers}

A prototype tool, SARDAIGNE, has been developed in SAS (Statistical Analyses Software, see www.sas.com), including a graphical interface for the user. It is based on the proposed methodology and is divided in four main parts:

- import and data management

- $\quad$ analyse for one simulation

- production of list of scenarios in order to test factors influence

- $\quad$ analyse of list of simulations

\section{Conclusions}

To conclude, timetable robustness, the capacity of a timetable to resist small perturbations, has to be estimated by simulation before applying modifications to the railway system (increase of traffic density, modification of signalling system, etc.). The methodology has to include a probabilistic/statistical view of perturbations of railway system.

In this paper, we described the main ideas of how to identify randomness, how to study robustness by simulation and how to evaluate a lack of precision in the results.

Some examples cases are given. A tool for providing such decision help has been developed.

\section{References}

[1] D. Benoist, Yves Tourbier and S. Germain-Tourbier, Plans d'expériences: construction et analyse, Technique \& documentation, Lavoisier, 1994.

[2] B. Efron, R. J. Tibshirani, An introduction to the bootstrap, Chapman \& Hall, 1993

[3] M. Fontaine and D. Gauyacq, SISYFE: a toolbox to simulate the railway network functioning for many purposes. Some cases of application, World Congress on Railway Research, 2001

[4] G.S. Fishman, Monte Carlo, Concepts, algorithms and applications, Springer series in operations research, Springer, 1996. 